\title{
Correlation Between Expression of the Cancer/Testis Antigen KK-LC-1 and Helicobacter pylori Infection in Gastric Cancer
}

\author{
TAKASHI FUKUYAMA ${ }^{1}$, NOBUE FUTAWATARI ${ }^{2,3}$, YOSHINOBU ICHIKI ${ }^{4}$, AKIKO SHIDA $^{3}$, \\ TAIGA YAMAZAKI ${ }^{1}$, YATSUSHI NISHI ${ }^{5}$, HIROSHI NONOGUCHI ${ }^{1}$, \\ YOSHIHITO TAKAHASHI ${ }^{3,5}$, HITOSHI YAMAZAKI $^{6}$ and NORITADA KOBAYASHI ${ }^{1}$ \\ ${ }^{1}$ Division of Biomedical Research, and Departments of ${ }^{5}$ Surgery, and ${ }^{6}$ Pathology, \\ Kitasato University Medical Center, Kitamoto, Japan; \\ ${ }^{2}$ Department of Surgery, Sagamihara National Hospital, Sagamihara, Japan; \\ ${ }^{3}$ Department of Surgery, School of Medicine, Kitasato University, Sagamihara, Japan; \\ ${ }^{4}$ Second Department of Surgery, School of Medicine, \\ University of Occupational and Environmental Health, Kitakyushu, Japan
}

\begin{abstract}
Background/Aim: Our previous study indicated that Kita-kyushu lung cancer antigen-1 (KK-LC-1) is a cancer/testis antigen (CTA) expressed in $82 \%$ of gastric cancer cases. Here, we investigated the relationship between $K K-L C-1$ expression and Helicobacter pylori infection in Japanese patients with gastric cancer. Patients and Methods: We examined CTA expression in 25 surgical gastric cancer specimens and anti-H. pylori IgGs in the serum of each patient. Results: KK-LC-1 was expressed in $80 \%$ of tumor samples, markedly higher than melanoma antigen gene (MAGE)-A1, MAGE-A3, MAGE-A4, synovial sarcoma, $X$ breakpoint 4 (SSX4) and New York esophageal squamous cell carcinoma-1 (NY-ESO-1). Anti-H. pylori IgG titers from the KK-LC-1-positive patients were significantly higher (67.5 7.6)

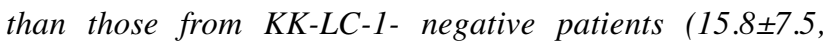
$p<0.01)$ although there were no significant differences between patients positive and negative for MAGE-A1, -A3 and-A4, SSX4 and NY-ESO-1. Conclusion: As far as we are aware, this is the first report of a correlation between a carcinogen and CTA expression in clinical samples. KK-LC-1 was frequently expressed in gastric cancer caused by $H$. pylori infection. The risk diagnosis for gastric cancer might be more accurate if KK-LC-1 expression status were also considered.
\end{abstract}

This article is freely accessible online.

Correspondence to: Dr. Takashi Fukuyama, Division of Biomedical Research, Kitasato University Medical Center, 6-100 Arai, Kitamoto, Saitama 364-8501, Japan. Tel: +81 485931236, Fax: +81 485931262, e-mail fukuyam@insti.kitasato-u.ac.jp.

Key Words: KK-LC-1, cancer/testis antigen, gastric cancer, Helicobacter pylori, carcinogen.
Several tumor-associated antigens have been identified in various human cancer types (1-3). These antigens are classified into four categories, excluding extrinsic viral antigens, as follows: cancer/testis antigens (CTAs), differentiation antigens, amplification or overexpression antigens, and tumor-specific mutated antigens. CTAs are especially attractive targets for immunotherapy because they are not, or are only minimally expressed in normal tissues except for testis but are aberrantly expressed in a range of human cancer types (4). Therefore, immunotargeting of these antigens is thought to have negligible adverse side-effects. Moreover, they could also be advantageous molecules for systemic diagnosis of cancer because of their specific expression pattern. However, data on the frequency of individual CTA expression is not currently sufficient for diagnostic applications.

We previously identified Kita-kyushu lung cancer antigen-1 (KK-LC-1) as a new CTA that has an epitope peptide recognized by cytotoxic T-lymphocytes (CTLs). KK-LC-1 maps to chromosome $\mathrm{Xq} 22$, is not expressed in normal tissues except for testis, and is expressed in $33 \%$ of non-small cell lung cancer $(5,6)$. Furthermore, we found that KK-LC-1 was expressed in $82 \%$ of gastric cancer (7). According to these data, KK-LC-1 could be categorized as a CTA, but its frequency in gastric cancer seems to depart from the traditional concept of CTAs.

In Japan, gastric cancer has the second highest incidence rate of all cancer, and more than $80 \%$ of cases are caused by Helicobacter pylori infection (8). We reported that $H$. pylori infection can induce expression of specific CTAs in addition to causing malignant transformation of host cells (9). These findings suggest that specific CTA expression might correlate with the initial cancer-causing event; therefore, CTAs could contribute as new candidates for immunotherapy and diagnosis of gastric cancer. 
In this study, we investigated the relationship between expression of KK-LC-1 and H. pylori infection in gastric cancer.

\section{Patients and Methods}

Human rights statement and informed consent. This study involved human participants. All procedures followed were in accordance with the ethical standards of Kitasato University Medical Center (approval no. 23-8) and with the Declaration of Helsinki. All patients, or their legal guardians, contributed in this study provided their written informed consent before this study.

Patients. A total of 46 patients underwent surgical resection for gastric cancer at the Department of Surgery, Kitasato University Medical Center, Kitamoto, Japan between August 2011 and August 2012. We obtained 25 sets of tumor samples and serum from these patients. The clinicopathological findings were classified according to the Japanese Classification of Gastric Carcinoma [14th edition (10)]. There is no bias in the background characteristics among the patients who participated in this study (Table I).

Tissue specimens. Each sample from resected tumor tissues was immediately preserved at $4^{\circ} \mathrm{C}$ overnight in RNAlater (Life Technologies, Carsbad, CA, USA), and then stored at $-80^{\circ} \mathrm{C}$ until used.

Expression of CTAs. Total RNA extraction from tumor samples, cDNA synthesis, the assay of gene expression for $\beta$-actin and each CTA was described elsewhere by us (7). The gene expression of $\beta$ actin; melanoma antigen gene (MAGE)-A1, -A3 and -A4; New York esophageal squamous cell carcinoma-1 (NY-ESO-1); and synovial sarcoma, X breakpoint 4 (SSX4) were measured with TaqMan Gene Expression Assays ID Hs99999903_m1, Hs00607097_m1, H200366532_m1, Hs00365979_m1, Hs00265824_m1 and Hs02341 532_m1, respectively, using a 7900HT Fast Real-Time PCR system (all Life Technologies, Carsbad, CA, USA). For cDNAs for which expression [represented by threshold cycle number $(\mathrm{Ct})]$ of the $\beta$-actin $(A C T B)$ gene yielded a Ct of $<28$, the expression of KK-LC-1 was examined using end-point reverse transcription polymerase chain reaction (RT-PCR) rather than a probe-based assay, since an appropriate probe for detecting KK-LC-1 mRNA has not been established. For RT-PCR of KK-LC-1, the oligonucleotides 5'ATGAACTTCT ATTTACTCCTAGCGAGC-3' and 5'-TTAGGT GGATTTCCGGTGAGG-3' (Sigma-Aldrich Japan, Shinagawa-ku, Japan) were used as specific primers, and annealing was performed at $67^{\circ} \mathrm{C}$ for 40 cycles, yielding a 342-bp product.

Evaluation of $H$. pylori-infection. There are many reports showing that $H$. pylori infection can be accurately measured by detecting anti- $H$. pylori antibodies in serum (11). We evaluated $H$. pylori infection using serum from the 25 gastric patients with cancer Serum anti-H. pylori $\mathrm{IgG}$ in was measured using a quantitative antiIgG H. pylori, ELISA Kit, (Phoenix Pharmaceuticals, Burlingame, CA, USA) according to the manufacturer's instructions.

Statistical analysis. Statistical analyses of anti- $H$. pylori IgG titers between positive and negative expression groups for each CTA were performed using Wilcoxon's rank-sum test. $p$-Values of less than 0.01 were considered significant; JMP8.0 (SAS institute Japan, Minato-ku, Japan) was used for the analysis.
Table I. Characteristics of 25 patients with gastric cancer.

\begin{tabular}{lc}
\hline & Value \\
\hline Mean age (range), years & $69.5(37-82)$ \\
Gender, n (\%) & \\
Male & $16(64.0 \%)$ \\
Female & $9(36.0 \%)$ \\
Depth of invasion, n (\%) & \\
T1 & $7(28.0 \%)$ \\
T2 & $6(24.0 \%)$ \\
T3 & $5(20.0 \%)$ \\
T4 & $7(28.0 \%)$ \\
Lymph node metastasis, n (\%) & \\
N0 & $11(44.0 \%)$ \\
N1 & $6(24.0 \%)$ \\
N2 & $4(16.0 \%)$ \\
N3 & $4(16.0 \%)$ \\
Histological type, n (\%) & \\
Papillary adenocarcinoma & $1(4.0 \%)$ \\
Tubular adenocarcinoma & $11(44.0 \%)$ \\
Mucinous adenocarcinoma & $1(4.0 \%)$ \\
Poorly differentiated adenocarcinoma & $11(44.0 \%)$ \\
Signet ring cell carcinoma & $1(4.0 \%)$ \\
Stage of disease, n (\%) & \\
I & $9(36.0 \%)$ \\
II & $10(40.0 \%)$ \\
III & $3(12.0 \%)$ \\
IV & $3(12.0 \%)$ \\
\hline & \\
&
\end{tabular}

\section{Results}

Expression of each CTA. We used reverse transcription polymerase chain reaction (RT-PCR) to determine the fraction of tumor samples from 25 patients that expressed specific CTAs. The expression profile of each CTA is shown in Table II. The frequency of expression of KK-LC-1 (80\%) was the highest among CTAs tested.

Correlation between CTA expression and $H$. pylori infection. We next divided the patients into positive- and negative-expression groups for each CTA and examined the difference in anti-H. pylori $\mathrm{IgG}$ titer for each group. When patients were divided for KK-LC-1 expression, the IgG titer was significantly higher in the positive group $(61.6 \pm 7.4 \mathrm{U} / \mathrm{ml})$ than in the negative one $(15.7 \pm 14.8 \mathrm{U} / \mathrm{ml}$, $p<0.01$, Table III and Figure 1A). Four out of the eight patients with IgG titer below $20 \mathrm{U} / \mathrm{ml}$ also expressed $\mathrm{KK}$ LC-1, while in the group with IgG titer above 20U/ml, 16 out of 17 (94\%) expressed KK-LC-1. No significant differences in anti-H. pylori IgG titer were found when the patients were grouped for expression of any other CTA (Table III and Figure 1B-F). 
Table II. The cancer/testis antigen (CTA) expression in 25 patients with gastric cancer.

\begin{tabular}{lccc}
\hline CTA & Negative, $n$ & Positive, $\mathrm{n}$ & $\begin{array}{c}\text { Frequency } \\
\text { of positivity (\%) }\end{array}$ \\
\hline KK-LC-1 & 5 & 20 & 80.0 \\
MAGE-A1 & 14 & 11 & 44.0 \\
MAGE-A3 & 13 & 12 & 48.0 \\
MAGE-A4 & 20 & 5 & 20.0 \\
NY-ESO-1 & 19 & 6 & 24.0 \\
SSX4 & 21 & 4 & 16.0 \\
\hline
\end{tabular}

KK-LC-1, Kitakyushu lung cancer antigen-1; MAGE, melanoma antigen gene; NY-ESO-1, New York esophageal squamous cell carcinoma-1; SSX4, synovial sarcoma, X breakpoint 4.

Table III. Anti-Helicobacter pylori IgG titer in each cancer/testis antigen (CTA) expression group.

\begin{tabular}{lccc}
\hline CTA & \multicolumn{2}{c}{ IgG titer (average \pm SE) } & \multirow{2}{*}{$p$-Value } \\
\cline { 2 - 3 } & Negative expression & Positive expression & \\
\hline KK-LC-1 & $15.7 \pm 14.8$ & $61.6 \pm 7.4$ & 0.0098 \\
MAGE-A1 & $59.6 \pm 10.0$ & $43.3 \pm 11.2$ & 0.2503 \\
MAGE-A3 & $60.9 \pm 10.3$ & $43.3 \pm 10.7$ & 0.2109 \\
MAGE-A4 & $48.3 \pm 8.3$ & $69.1 \pm 16.7$ & 0.3415 \\
NY-ESO-1 & $56.8 \pm 8.6$ & $38.8 \pm 15.3$ & 0.2794 \\
SSX4 & $49.5 \pm 8.2$ & $68.1 \pm 18.8$ & 0.4149 \\
\hline
\end{tabular}

KK-LC-1, Kitakyushu lung cancer antigen-1; MAGE, melanoma antigen gene; NY-ESO-1, New York esophageal squamous cell carcinoma-1; SSX4, synovial sarcoma, X breakpoint 4 .

\section{Discussion}

CTAs are expressed in about half of all tumors from a wide range of different organs such as melanoma, lung, esophagus, gastric, colon and breast carcinoma, but not in normal tissues except for germline tissues. KK-LC-1 is a CTA by this definition, because it is not expressed in normal tissues except for testis and cancer of multiple organs; however, it is expressed in $80 \%$ of gastric cancer cases, which is exceedingly more frequent than has been observed for other CTAs in other cancer types (7). To resolve this aberration, we confirmed the correlation of KK-LC-1 expression and $H$. pylori infection, which is the major carcinogenic factor for gastric cancer in Japan. There are a few reports that suggest that tumorigenic factors induced or enhanced the expression of specific CTAs $(9,12)$. However, as far as we are aware, this is the first report linking
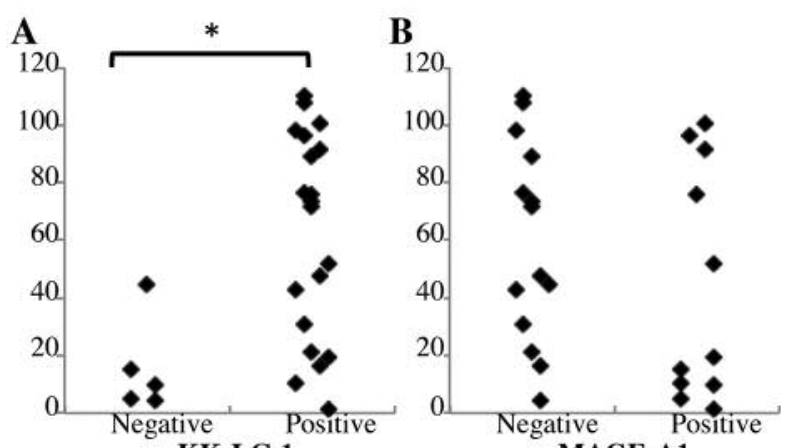

KK-LC-1

MAGE-A1
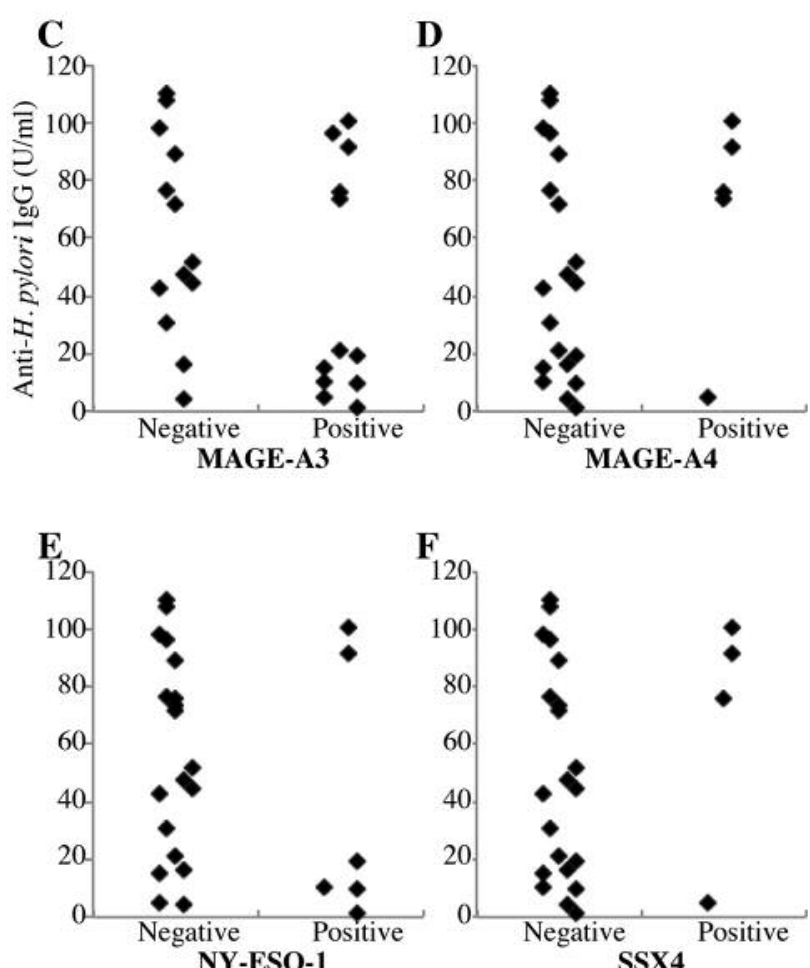

Figure 1. Relationship between anti-Helicobacter pylori IgG and cancer/testis antigen (CTA) expression in gastric cancer. Patients were divided into positive or negative groups for expression of each CTA: A: Kitakyushu lung cancer antigen-1 (KK-LC-1), B: melanoma antigen gene (MAGE)-A1, C: MAGE-A3, D: MAGE-A4, E: New York esophageal squamous cell carcinoma-1(NY-ESO-1) and F: synovial sarcoma, X breakpoint 4 (SSX4). *p<0.01 using Wilcoxon rank-sum test.

carcinogen exposure to CTA expression in a clinical setting. In this study, we found that $H$. pylori infection was correlated with KK-LC-1-expression. These data might indicate that each CTA is induced by a specific individual or group of carcinogens. Additionally, it might be possible that the cause of cancerization could be clarified by analyzing CTA expression. 
We assessed $H$. pylori infection using anti-H. pylori IgG in serum because this assay gives an accurate reflection of the infection status of the patient (11). Following the manufacture's protocol, a reading below 20 $\mathrm{U} / \mathrm{ml}$ was considered negative for $H$. pylori infection. From our patients, four out of eight $(50 \%)$ cases where the value was below $20 \mathrm{U} / \mathrm{ml}$ expressed KK-LC-1. The frequency of $\mathrm{KK}-\mathrm{LC}$-1-expression in this group was similar to that of other CTAs (Table III) $(13,14)$. In contrast, 16 out of 17 (94\%) patients with titers over 20 $\mathrm{U} / \mathrm{ml}$ were positive for $\mathrm{KK}-\mathrm{LC}-1$ expression. These results suggest that $H$. pylori infection might induce tumor cells to express KK-LC-1. In only one case was KK-LC-1 not expressed despite a high IgG titer against $H$. pylori. This might be due to the $10 \%$ inaccuracy rate of ELISA (11) or to the diversity of $H$. pylori; $90 \%$ of the H.pylori carried in the Japanese population is the East Asia type which has a strong potential for carcinogenesis (15).

CTAs are attractive candidates for diagnosing cancer even if their application for cancer immunotherapy is limited. KK-LC-1 may be a good candidate to help diagnose gastric cancer because of its high frequency of expression and specificity for tumors involving $H$. pylori infection. There is currently a risk of gastric cancer designated as the $\mathrm{ABC}(\mathrm{D})$ classification, which stratifies patients into three or four risk groups using $H$. pylori infection and atrophic gastritis by measuring anti-H. pylori $\mathrm{IgG}$ and pepsinogen I/II in serum (16). However, it is difficult to detect patients with gastric cancer even in the high-risk group (high $H$. pylori titer and low pepsinogen, and high or low H. pylori titer and high pepsinogen) because fewer than $3 \%$ of the tumors are detected (17). Mining KK-LC-1 might enable the high-risk group to be narrowed-down and the detection rate increased. We detected KK-LC-1 by gene expression using gastric cancer specimens. Therefore, this diagnostic assay needs to be accompanied by an invasive operation. Our future work hopes to establish a less invasive system for detect KK-LC-1. Adding KK-LC-1 detection to H. pylori and pepsinogen measurements might establish a highly accurate diagnosis of gastric cancer.

\section{Acknowledgements}

T. Fukuyama acknowledges support from the JSPS, KAKENHI (21700510), Takeda Science Foundation, a Kitasato University Research Grant for Young Researchers and Grant-in-Aid for research by Kitasato University Medical Center (H25-0006). T. Yamazaki acknowledges support from a Kitasato University Research Grant for Young Researchers.

The Authors thank Dr. Takayuki Uematsu, Ms. Rui Yamamura, Ms. Etsuko Terayama, Ms. Maki Kobayashi and Mr. Yukio Nishi for their technical assistance, and also thank Mr. James Mahaffey of Edanz group Japan and Atsuko Fujisawa for English proofreading.

\section{References}

1 van der Bruggen $P$, Traversari $C$, Chomez $P$, Lurquin $C$, De Plaen E, Van den Eynde B, Knuth A and Boon T: A gene encoding an antigen recognized by cytolytic T-lymphocytes on a human melanoma. Science 254: 1643-1647, 1991.

2 Ichiki Y, Takenoyama M, Mizukami M, So T, Sugaya M, Yasuda M, So T, Hanagiri T, Sugio $K$ and Yasumoto $K$ : Simultaneous cellular and humoral immune response against mutated p53 in a patient with lung cancer. J Immunol 172: 4844-4850, 2004.

3 Takenoyama M, Baurain JF, Yasuda M, So T, Sugaya M, Hanagiri T, Sugio K, Yasumoto K, Boon T and Coulie PG: A point mutation in the $N F Y C$ gene generates an antigenic peptide recognized by autologous cytolytyic T-lymphocytes on a human squamous cell lung carcinoma. Int J Cancer 118: 1992-1997, 2006.

4 Scanlan MJ, Simpson AJ and Old LJ: The cancer/testis genes: standardization, and commentary. Cancer Immun 4: 1, 2004.

5 Fukuyama T, Hanagiri T, Takenoyama M, Ichiki Y, Mizukami $M$, So T, Sugaya $M$, So $T$, Sugio $K$ and Yasumoto $K$ : Identification of a new cancer/germline gene, KK-LC-1, encoding an antigen recognized by autologous CTL induced on human lung adenocarcinoma. Cancer Res 66: 4922-4928, 2006.

6 Shigematsu Y, Hanagiri T, Shiota H, Kuroda K, Baba T, Mizukami M, So T, Ichiki Y, Yasuda M, So T, Takenoyama M and Yasumoto $\mathrm{K}$ : Clinical significance of cancer/testis antigens expression in patients with non-small cell lung cancer. Lung Cancer 68: 105-110, 2010.

7 Shida A, Futawatari N, Fukuyama T, Ichiki Y, Takahashi Y, Nishi Y, Kobayashi N, Yamazaki H and Watanabe M: Frequent high expression of kita-kyushu lung cancer antigen-1 (KK-LC-1) in gastric cancer. Anticancer Res 35: 3575-3579, 2015.

8 Uemura N, Okamoto S, Yamamoto S, Matsumura N, Yamaguchi S, Yamakido M Taniyama K, Sasaki N and Schlemper RJ: Helicobacter pylori infection and the development of gastric cancer. N Engl J Med 345: 784-789, 2001.

9 Fukuyama T, Yamazaki T, Fujita T, Uematsu T, Ichiki Y, Kaneko $\mathrm{H}$, Suzuki $\mathrm{T}$ and Kobayashi $\mathrm{N}$ : Helicobacter pylori, a carcinogen, induces the expression of melanoma antigenencoding gene (MAGE)-A3, a cancer/testis antigen. Tumor Biol 33: 1881-1887, 2012.

10 Japanese Gastric Cancer Association: Japanese Classification of Gastric Carcinoma: Third English Edition. Gastric Cancer 14: 101-112, 2011.

11 Rahman SH, Azam MG, Rahman MA, Arfin MS, Alam MM, Bhuiyan TM, Ahmed N, Rahman M, Nahar S and Hassan MS: Non-invasive diagnosis of $H$. pylori infection: evaluation of serological tests with and without current infection marker CIM. World J Gastroenterol 14: 1231-1236, 2008.

12 Sharma A, Bode B, Wenger RH, Lehmann K, Sartori AA, Moch $\mathrm{H}$, Knuth A, von Boehmer L and van den Broek M: $\gamma$-Radiation promotes immunological recognition of cancer cells through increased expression of cancer-testis antigens in vitro and in vivo. PLoS One 6: e28217, 2011.

13 Wang Y, Wu XJ, Zhao AL, Yuan YH, Chen YT, Jungbluth AA, Gnjatic S, Santiago D, Ritter G, Chen WF, Old LJ and Ji JF: Cancer/testis antigen expression and autologous humoral immunity to NY-ESO-1 in gastric cancer. Cancer Immun 4: 11, 2004. 
14 Nakamura Y, Tanaka F, Nagahara H, Ieta K, Haraguchi N, Mimori K, Sasaki A, Inoue H, Yanaga K and Mori M: OPAinteracting protein $5($ OIP5) is a novel cancer-testis specific gene in gastric cancer. Ann Surg Oncol 14: 885-892, 2007.

15 Tartaglia M, Niemeyer CM, Fragale A, Song X, Buechner J, Jung A, Hahlen K, Hasle H, Licht JD and Gelb BD: Somatic mutations in PTPN11 in juvenile myelomonocytic leukemia, myelodysplastic syndromes and acute myeloid leukemia. Nat Genet 34: 148-150, 2003.

16 Itoh T, Saito M, Marugami N, Hirai T, Marugami A, Takahama $\mathrm{J}$, Tanaka $\mathrm{T}$ and Kichikawa $\mathrm{K}$ : Correlation between the ABC classification and radiological findings for assessing gastric cancer risk. Jpn J Radiol 33: 636-644, 2015.
17 Mizuno S, Miki I, Ishida T, Yoshida M, Onoyama M, Azuma T, Habu Y, Inokuchi $\mathrm{H}$, Ozasa K, Miki $\mathrm{K}$ and Watanabe Y: Prescreening of a high-risk group for gastric cancer by serologically determined Helicobacter pylori infection and atrophic gastritis. Dig Dis Sci 55: 3132-3137, 2010.

Received February 14, 2017

Revised March 23, 2017

Accepted March 24, 2017 